\section{A Novel Virtual Reality Technique (Cervigame®) Compared to Conventional Proprioceptive Training to Treat Neck Pain: A Randomized Controlled Trial}

\author{
Rezaei I. ${ }^{1,}$, Razeghi M. ${ }^{1,3 *}{ }^{\oplus}$, Ebrahimi S. ${ }^{1,2}$, Kayedi S., ${ }^{1,}$ \\ Rezaeian Zadeh A. ${ }^{4}$
}

\begin{abstract}
Background: Despite the potential benefits of virtual reality technology in physical rehabilitation, only a few studies have evaluated the efficacy of this type of treatment in patients with neck pain.

Objective: The aim of this study was to compare the effects of virtual reality training (VRT) versus conventional proprioceptive training (CPT) in patients with neck pain.

Materials and Methods: Forty four participants with nonspecific chronic neck pain were randomly assigned to VRT or CPT in this assessor-blinded clinical trial. A novel videogame called Cervigame ${ }^{\circledR}$ was designed for VRT. It comprises of 50 stages divided into unidirectional and two-directional stages ordered from easy to hard. CPT consisted of eye-follow, gaze stability, eye-head coordination and position and movement sense training. Both groups completed 8 training sessions over 4 weeks. Visual analogue scale score, neck disability index and Y-balance test results were recorded at baseline, immediately after and 5 weeks post-intervention. Mixed repeated measure ANOVA was used to analyze differences between mean values for each variable at an alpha level of 0.05 .
\end{abstract}

Results: There were significant improvements in all variables in both groups immediately after and 5 weeks after the intervention. Greater improvements were observed in the visual analogue scale and neck disability index scores in VRT group, and the results for all directions in Y-balance test were similar in both groups. No side effects were reported.

Conclusion: Improvements in neck pain and disability were greater in VRT than CPT group. Cervigame ${ }^{\circledR}$ is a potentially practical tool for rehabilitation in patients with neck pain.

Citation: Rezaei I, Razeghi M, Ebrahimi S, Kayedi S, Rezaeian Zadeh A. A Novel Virtual Reality Technique (Cervigame®) Compared to Conventional Proprioceptive Training to Treat Neck Pain: A Randomized Controlled Trial. J Biomed Phys Eng. 2019;9(3):355-366. https://doi org/10.31661/jbpe.v0i0.556.

\section{Keywords}

Neck Pain, Virtual Reality, Proprioception, Cervicocephalic Kinesthesia, Rehabilitation, Physical Therapy

\section{Introduction}

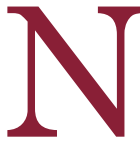
eck pain (NP) is a global problem [1], with a reported lifetime prevalence of about $70 \%$ in different populations $[2,3]$. $\mathrm{Pa}-$ tients with NP have demonstrated impairment in a wide range of sensorimotor functions [4-7]. The density of mechanoreceptors and muscle spindles in cervical region has increased $[8,9]$, and these elements are associated with vestibular, visual and postural control systems
${ }^{1}$ Department of Physical Therapy, School of Rehabilitation Sciences, Shi-

raz University of Medical

Sciences, Shiraz, Iran

${ }^{2}$ Student Research Committee, Shiraz University of Medical Sciences,

Shiraz, Iran

${ }^{3}$ Rehabilitation Sciences Research Center, Shiraz University of Medical Sciences, Shiraz, Iran

${ }^{4}$ Department of Epi-

demiology, School of

Health, Shiraz University

of Medical Sciences,

Shiraz, Iran

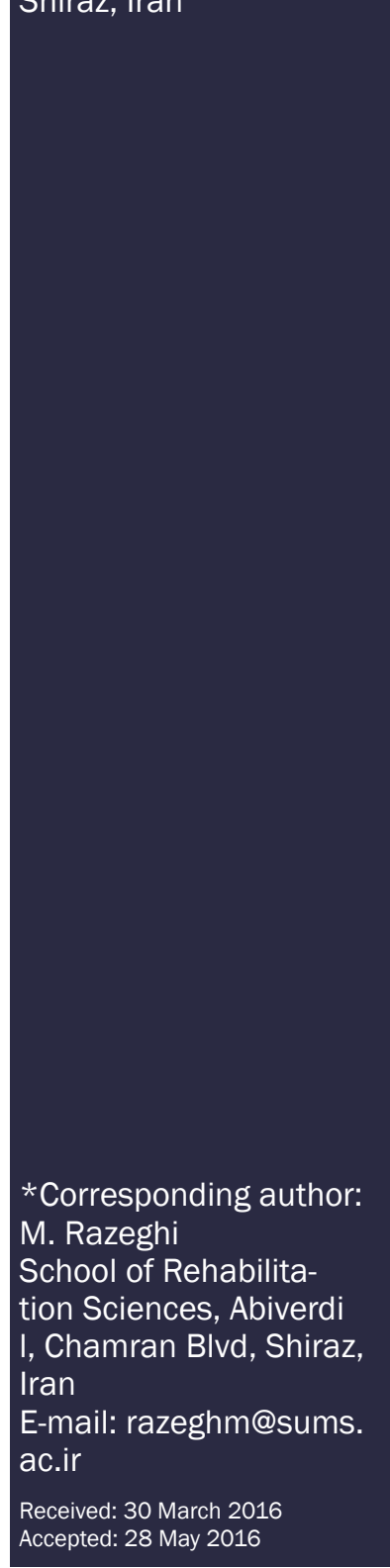

Accepted: $28 \mathrm{Mmy} 2$ 
which provide information that is important in regulating postural balance $[10,11]$. Evidence to date supports the important role of cervical sensory input for postural control [12]. Patients with NP have impaired balance compared to healthy controls [13], and because of the prominent function of cervical proprioception, different sensorimotor training protocols have been tested for rehabilitation in patients with NP [14-17].

Virtual reality (VR) is a term that describes innovative, real-time computer-based technologies that play an increasing role in the field of physical rehabilitation for many groups of patients [18, 19]. Recently, a VR approach was used to reduce pain in a psychological intervention [20]. This type of intervention is known as a distraction technique, because it draws attention away from pain toward pleasant sensory stimuli [21]. Virtual reality has also been shown to be effective in reducing pain and discomfort in patients with different types of chronic pain [20-24]. Unlike conventional rehabilitation, motivation and interactivity are the main practical benefits of VR training (VRT), especially in video gamebased approaches to therapy [25]. Patients play the therapeutic game repeatedly to gain a better score without becoming bored [25]. Feedback, an essential component of motor learning, is a prominent feature in virtual environments [19].

Despite potential benefits of VR technology in physical rehabilitation [19] and pain reduction [20-24], only a few studies have evaluated the efficacy of this type of treatment in patients with NP $[26,27]$. Recently, a VR device was developed to assess [7] and treat [26] cervical kinematics in patients with NP. In a pilot study to compare the effects of cervical kinematic training with and without this new VR device, between-group analysis showed improvements in overall perceived change in VR plus kinematic training group but no significant improvement in neck disability, range of motion or dynamic balance [26].
The present study investigated a new gamebased virtualized reality tool called Cervigame ${ }^{\circledR}$, designed to reduce pain and improve prorioceptive function in patients with NP. The aim of the study was to compare the effects of VRT versus conventional proprioceptive training (CPT) on NP, functional disability and postural control in patients with nonspecific chronic NP. In addition, this study explored the clinical applicability and user experience of training with Cervigame ${ }^{\circledR}$. In light of the advantages (noted above) of virtual versus conventional rehabilitation, we hypothesized that video game-based training is more effective than conventional proprioceptive exercises in reducing pain and disability, and in enhancing balance in patients with chronic NP.

\section{Material and Methods}

\section{Participants}

The inclusion criteria for patients who took part in this study were a history of nontraumatic NP for more than 3 months and age between 20 and 55 years. They were recruited by distributing leaflets in public places of the city.

Exclusion criteria were a score $\geq 15$ and $\leq 9$ (out of possible 50) on Neck Disability Index (NDI), history of cervical and thoracic trauma within the 6 months before examination, neurological signs and symptoms in the upper extremities, nerve injury, spinal cord compression, cervical spine pathology or surgery and cancer.

Ethical approval for the study was provided by the institutional medical research ethics committee (Code: CT-92-6895). The participants provided their written consent before participation.

\section{Study Design}

This study was a randomized clinical trial that compared two exercise protocols immediately after intervention and 5 weeks postintervention. It was registered in the Iranian Registry of Clinical Trials (IRCT registration 
number IRCT2014040817177N1). Patients were randomly assigned to one of two exercise groups (CPT or VRT) with a computer-generated sequence. Both protocols involved 2 sessions of training per week during 4 weeks, for a total of 8 training sessions. All sessions took place under the supervision of an experienced physical therapist.

Each session lasted $21 \mathrm{~min}$ in both groups. Initially, there were 5 min of warm-up exercises with active cervical and scapular range of motion in 3 planes. After that, there was 1 min of exercise training followed by a $1-\mathrm{min}$ rest, and this sequence was repeated 8 times.

CPT

The exercise protocol was based on previous studies $[15,16]$. Exercises consisted of eye-follow, gaze stability, eye-head coordination and position sense and movement sense practice.

In the eye-follow exercise, patients moved their eyes to follow the target while seated with their head stationary. The target was a pen held by a physical therapist, who initially moved it slowly in one plane and then increased the speed and changed the direction of movement. For the gaze stability exercise, patients actively moved their head in all directions while visually fixing on the target.

The exercise for eye-head coordination began by moving the head and eyes to the same side. Then participants moved their eyes first to keep focused on the target, and then moved their head. Finally, they moved their eyes in one direction while simultaneously rotating their head in the opposite direction. These exercises were initially done slowly in a restricted range of movements, then the speed and range of movements gradually increased. Exercises were done in both vertical and horizontal directions.

For joint position sense and movement sense exercises, participants wore a laser pointer attached to a headband. The patients sat 1 meter from a point marked on the wall, and were instructed to move their head until the laser beam was aimed on the point, and then to close their eyes and memorize their head-neck position for $5 \mathrm{~s}$. Maximal movement of the head was performed in one direction (flexion, extension, rotation or lateral flexion), after which the patients tried to recover their initial head position as closely as possible, and opened their eyes. The relocation error indicated by the distance of the laser beam from the point marked on the wall was used as feedback. Movement sense was practiced by using the head-mounted laser pointer to trace a moving object held by the physical therapist. The task was progressed by increasing the speed and changing the pattern of movement.

\section{VRT}

A new video game (Cervigame ${ }^{\circledR}$ version 1.01) was designed for training. The patients were seated $70 \mathrm{~cm}$ directly in front of a monitor screen. A reflective marker was affixed between and slightly above the patient's eyebrows. A Head Mouse Extreme ${ }^{\circledR}$ (Origin Instruments Corporation, Grand Prairie, Texas, USA) was placed above the laptop computer monitor screen. The head mouse was aimed on reflective marker, which represented head movements to control pointer movement on the laptop computer. The head mouse measurement rate was $45 \mathrm{~Hz}$ nominal with a 10 ms latency.

Cervigame ${ }^{\circledR}$ consists of 50 stages, each with a unique invisible movement pattern. The main visual component of the game is a rabbit attempting to reach carrots. The virtual carrots appear continuously along the line of the movement pattern being trained. This avatar is controlled by the patient's head movements. The more carrots the rabbit obtains, the higher the patient's score. Stages increase in difficulty as obstacles (e.g. mushrooms, trees, stones, etc.) appear in predefined positions. The rabbit must avoid colliding with these obstacles to obtain higher scores. The best score in each stage was obtained by capturing all carrots without colliding with any obstacles. The patients could obtain bonus stars based on 
their performance: 0 (below $50 \%$ of the highest score), 1 (between $50 \%$ and $59.99 \%$ of the highest score), 2 (between $60 \%$ and $74.99 \%$ of the highest score), 3 (between $75 \%$ and $89.99 \%$ of the highest score), 4 (between $90 \%$ and $99.99 \%$ of the highest score) or $5(100 \%$ of the highest score). If the score was below $50 \%$ of the highest possible score, no star was awarded, and the stage was repeated.

Based on the patient's head movement (along a line or in a plane) the stages are divided into two main categories: unidirectional and two-directional. The unidirectional category consists of 24 stages. The rabbit moves at constant speed in one direction, and its movement in the opposite direction is controlled by the patient's head movement. Twelve stages are designed to improve right and left cervical rotation (Figure 1a) and other 12 stages are designed to improve cervical flexion and extension (Figure 1b). The order of stages alternates as the game progresses.

The two-directional category consists of 26 stages. The rabbit's movement in all directions is controlled by patient's head movements. In these stages up to 7 carrots may be displayed sequentially according to the pattern of movement. When the next carrot appears, the previously displayed carrot disappears. The sequence is repeated until the end of the stage (Figure 1c).

The order of 50 stages progresses from easy to hard based on the stage category, number and acuity of the angles, shape and variation in the range of trajectories and the arrangement of obstacles.

In the first 3 treatment sessions, the patients play only unidirectional stages. Combinations of unidirectional and two-directional stages are played alternately in the fourth and fifth sessions. In the last 3 sessions, only two-directional stages are played. At the start of each session, the last two stages in the previous training session are played initially for accommodation.

\section{Outcome Measures}

Outcome measures were collected before and immediately after the initial interventions and 5 weeks post-intervention. The primary outcome measures were patient-reported NP and disability, and the secondary outcome was dynamic balance. Two examiners who were blinded to the patient group assignment recorded the outcome measures. Each patient was examined at 3 assessment times by the same examiner.

\section{Visual Analogue Scale (VAS)}

Average NP intensity during the last week was indicated by placing a mark on a $0-100$ mm VAS anchored with "no pain" at one end and "worst pain imaginable" at the other end. A minimal clinically-important change (MCIC) was defined as a difference of at least $25 \mathrm{~mm}$ between successive recordings of selfreported pain [28].

\section{Neck Disability Index}

This questionnaire was used to record selfrated disability in patients with NP [29]. NDI consists of 10 items, each rated on a scale from 0 (no disability) to 5 (complete disabil-
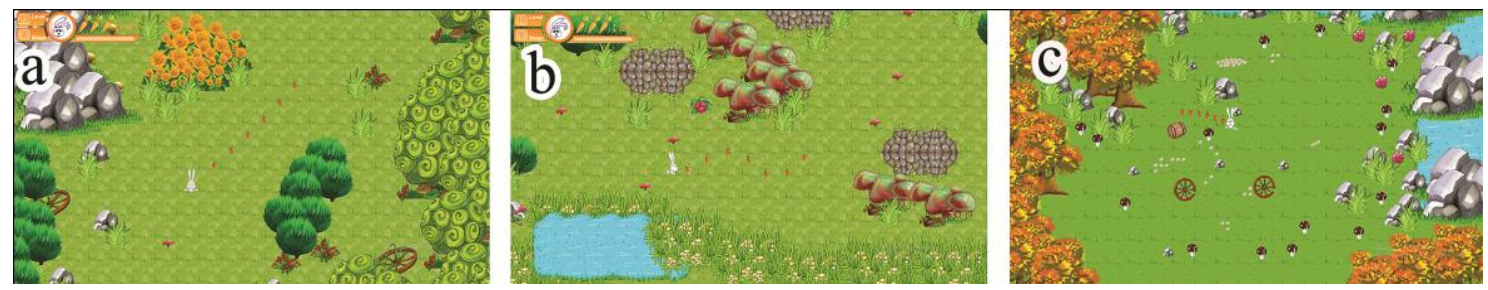

Figure 1: Screens showing examples of unidirectional and two-directional stages of the game. (a) A unidirectional screen for cervical right and left rotation. (b) A unidirectional screen for flexion and extension. (c) A two-directional screen. 
ity). The Iranian version of NDI, which was shown to be a reliable and valid questionnaire for Persian-speaking patients [30], was used in this study. The MCIC for NDI was defined as a change in score of 3.5 (out of possible 50) [28].

\section{Y-balance Test (YBT)}

For this test, 3 strips of tape approximately $150 \mathrm{~cm}$ long were affixed to the laboratory floor to form a Y-shaped pattern. One strip was placed first to form the anterior-facing "stem", and 2 additional strips were placed from one end of the first strip backward to form a symmetrical 135-degree angle (the "arms" of Yshape) marking the posteromedial (PM) and posterolateral (PL) directions.

Patients placed the heel of their dominant foot (the foot used to kick a ball) on the center of the pattern with their first toe aligned with the anterior-projecting line. They were instructed to reach backwards as far as they could with their nondominant leg along each arm of the Y-shape with their hands on their hips. They touched the tapes lightly as far as they could reach with the distalmost aspect of their first toe and returned to a double-leg stance while maintaining their balance.

The distance from the center of the pattern to the farthest point patients were able to reach with their toe was marked and recorded by the examiner. Trials were discarded and repeated if the stance foot moved or lifted from the floor, if the reach foot failed to return to the starting position or was used for support, or if hands were removed from the hips. The patients performed 4 practice trials, followed by test trials in anterior (ANT), PM and PL directions. The average of 3 reach distances in each direction was normalized by the individual's leg length as the distance from anterior superior iliac spine to the distal tip of the medial malleolus, and then multiplied by 100 . Composite reach distance was recorded as the average of 3 normalized reach directions multiplied by 100 .
Sample Size and Statistical Analysis

Sample size was calculated based on the comparison of mean scores on a VAS between groups in a previous study [31]. For an alpha error of $5 \%, 80 \%$ power and 0.8 effect size, 18 participants in each group were estimated. In anticipation of a $20 \%$ drop-out rate, the sample size increased to 22 participants in each group.

A multiple imputation technique was used to deal with missing data for participants who defaulted from the 5 -week post-intervention measurement. A $2 \times 3$ mixed repeated measure ANOVA with one between-subject factor (VRT, CPT) and one within-subject factor (time) was used to analyze differences between mean values for all variables at an alpha level of 0.05 . All statistical analyses were done with SPSS version 20.0 for Windows.

\section{Results}

Forty four patients with chronic NP participated in this study. One participant in VRT group did not complete the training sessions due to lack of time to attend. Another participant did not return for the follow-up assessment session because of a change in place of residence out of town. One participant in VRT group only completed the pain and disability tests but not the balance measures due to right ankle joint pain. In CPT group, one participant did complete the training sessions because of a positive laboratory pregnancy test, and one did not attend the 5-week follow-up session due to dissatisfaction with the treatment effect (Figure 2). Patients' characteristics in each group are summarized in Table 1.

\section{Neck Pain and Self-Rated Disabil- ity Measures \\ VAS}

There were significant main effects for time $\left(\mathrm{F}_{2.80}=167.16, \mathrm{P}<0.001\right)$ but not group $\left(\mathrm{F}_{1,40}=2.46, \mathrm{P}=0.124\right)$ and the interaction between time and group was significant $\left(\mathrm{F}_{2,80}=17.21, \mathrm{P}<0.001\right)$. After the intervention, 


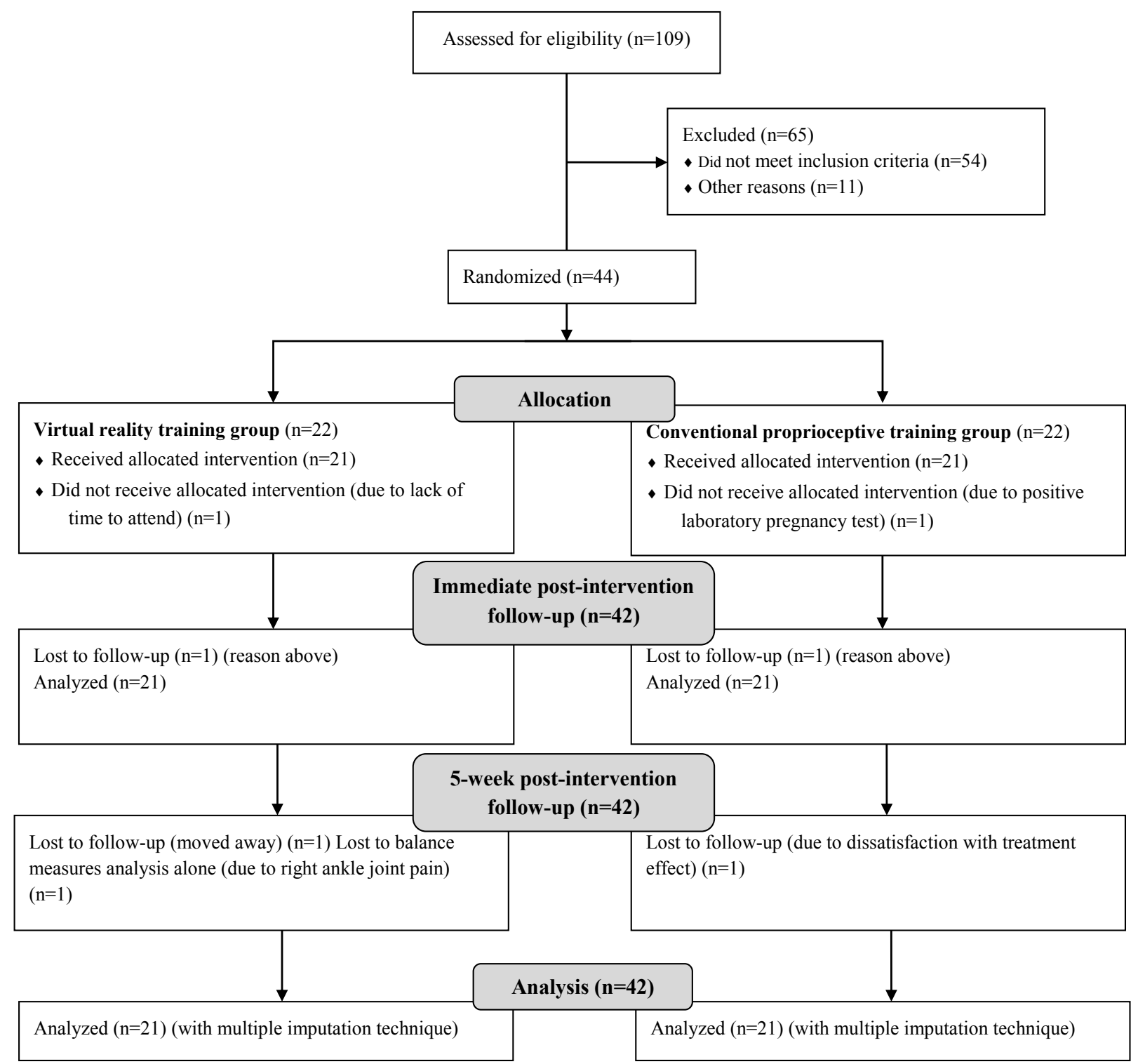

Figure 2: Flow diagram showing participant flow and follow-up evaluation.

VAS score improved by a mean of $36.36 \mathrm{~mm}$ in the VRT group and $19.32 \mathrm{~mm}$ in the CPT group. At the 5-week follow-up appointment, the improvements were $37.54 \mathrm{~mm}$ and 18.78 $\mathrm{mm}$, respectively (Figure 3a).

NDI

The main effects for both time $\left(\mathrm{F}_{2,80}=102.71\right.$, $\mathrm{P}<0.001)$ and group $\left(\mathrm{F}_{1,40}=17.70, \mathrm{P}<0.001\right)$ as well as the time $\times$ group interaction $\left(\mathrm{F}_{2,80}=17.71, \mathrm{P}<0.001\right)$ were all significant. NDI scores decreased by a mean of 8.43 af- ter VRT and 8.62 after 5 weeks. The reduction in NDI scores in the CPT group were less than half that in the VRT group immediately after the intervention (4.14) and after 5 weeks (3.06) (Figure 3b).

\section{Dynamic Balance Measures YBT}

The results of mixed repeated measure ANOVA were similar for all directions and the composite value of YBT. There were 
Table 1: Baseline demographic and clinical characteristics in each group

Variables

Age (years)

Sex (female \%)

Height (centimeters)

Body mass index

Duration of neck pain (months)

Visual analogue scale (100 millimeters)

Neck Disability Index (50)

Y-balance test normalized reach distance (centimeters)

Anterior direction

Posteromedial direction

Posterolateral direction

Composite value
VRT group $(\mathbf{n}=\mathbf{2 1})$

$36.19(9.80)$

42.90

$168.47(8.46)$

$24.32(4.21)$

$22.42(15.52)$

$47.11(10.24)$

$13.00(1.30)$

$97.30(5.53)$

$74.65(7.63)$

$64.02(6.87)$

$78.65(5.37)$
CPT group $(n=21)$

$31.23(9.49)$

52.4

$166.71(11.13)$

$24.12(4.91)$

$22.04(16.79)$

$38.95(10.07)$

$12.28(1.38)$

$97.66(8.15)$

$78.21(9.89)$

$68.77(11.36)$

$81.55(8.76)$

Abbreviations: VRT, virtual reality training; CPT, conventional proprioceptive training Values are the mean and (SD) or \%
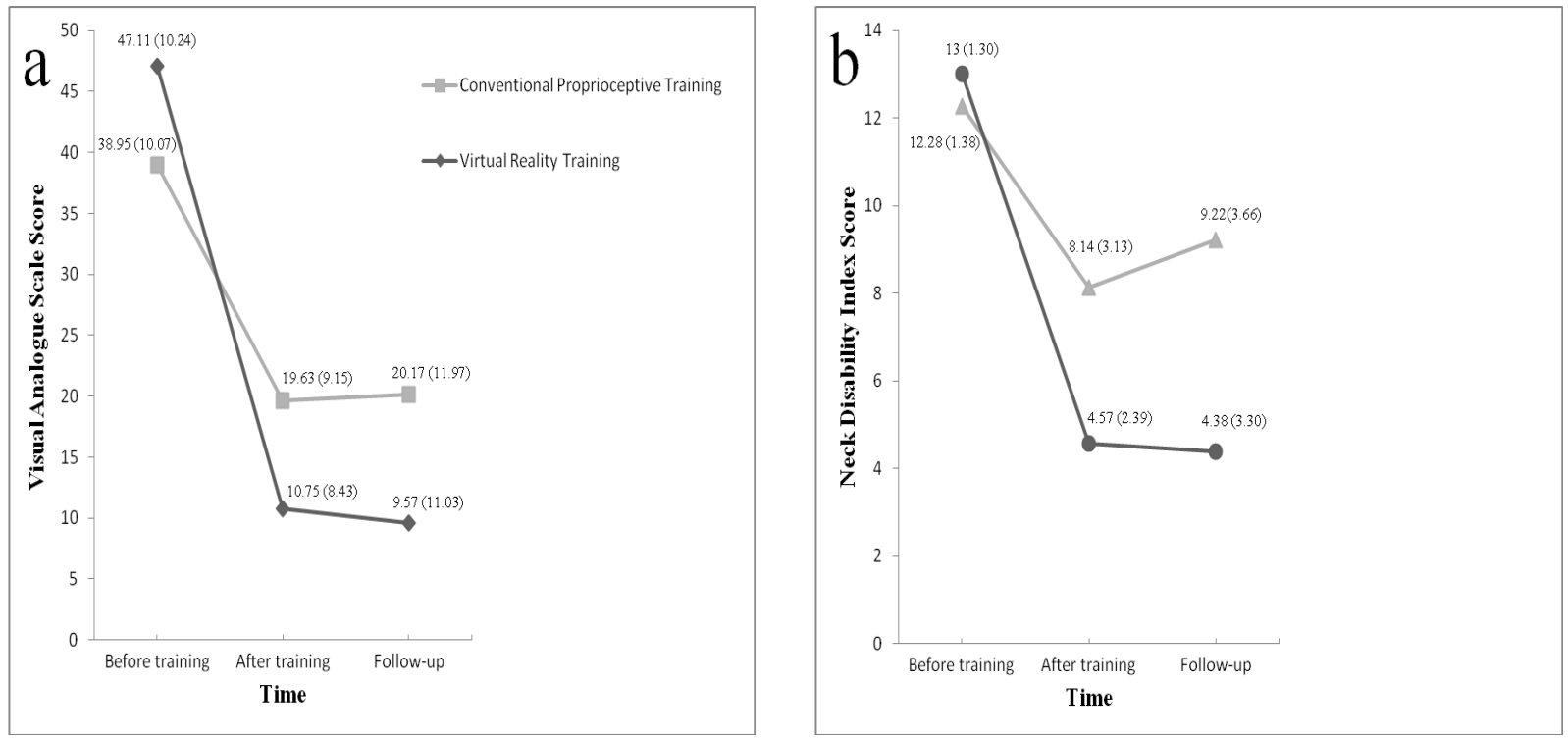

Figure 3: Group comparison of changes in (a) pain and (b) functional disability. Values are the mean and (SD) 
significant main effects of time for ANT $\left(\mathrm{F}_{1.64,65.94}=14.45, \mathrm{P}<0.001\right), \mathrm{PM}\left(\mathrm{F}_{2,80}=24.45\right.$, $\mathrm{P}<0.001)$, PL $\left(\mathrm{F}_{1.72,69.02}=28.86, \mathrm{P}<0.001\right)$ directions and composite value $\left(\mathrm{F}_{1.58,63.27}=31.43\right.$, $\mathrm{P}<0.001)$. No statistically significant interactions between time and group, nor significant main effects of group, were observed in any of the three directions or in the composite value
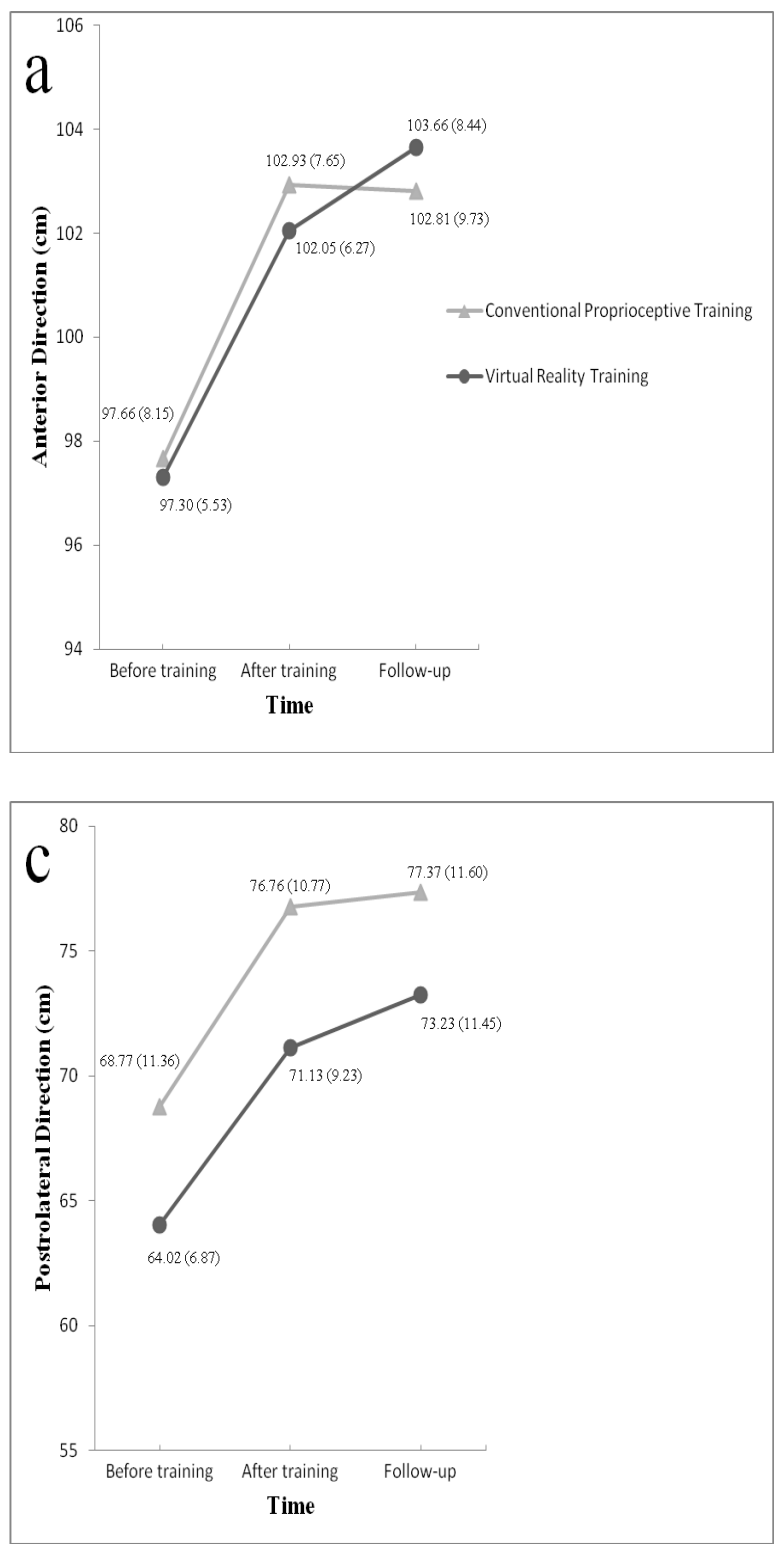

(Figure 4).

\section{Discussion}

This study presents a novel method of cervicocephalic kinesthetic training in the form of VR exercises used in the game we call Cervigame ${ }^{\circledR}$. The results indicated that both VRT and CPT protocols led to reduced pain and dis-
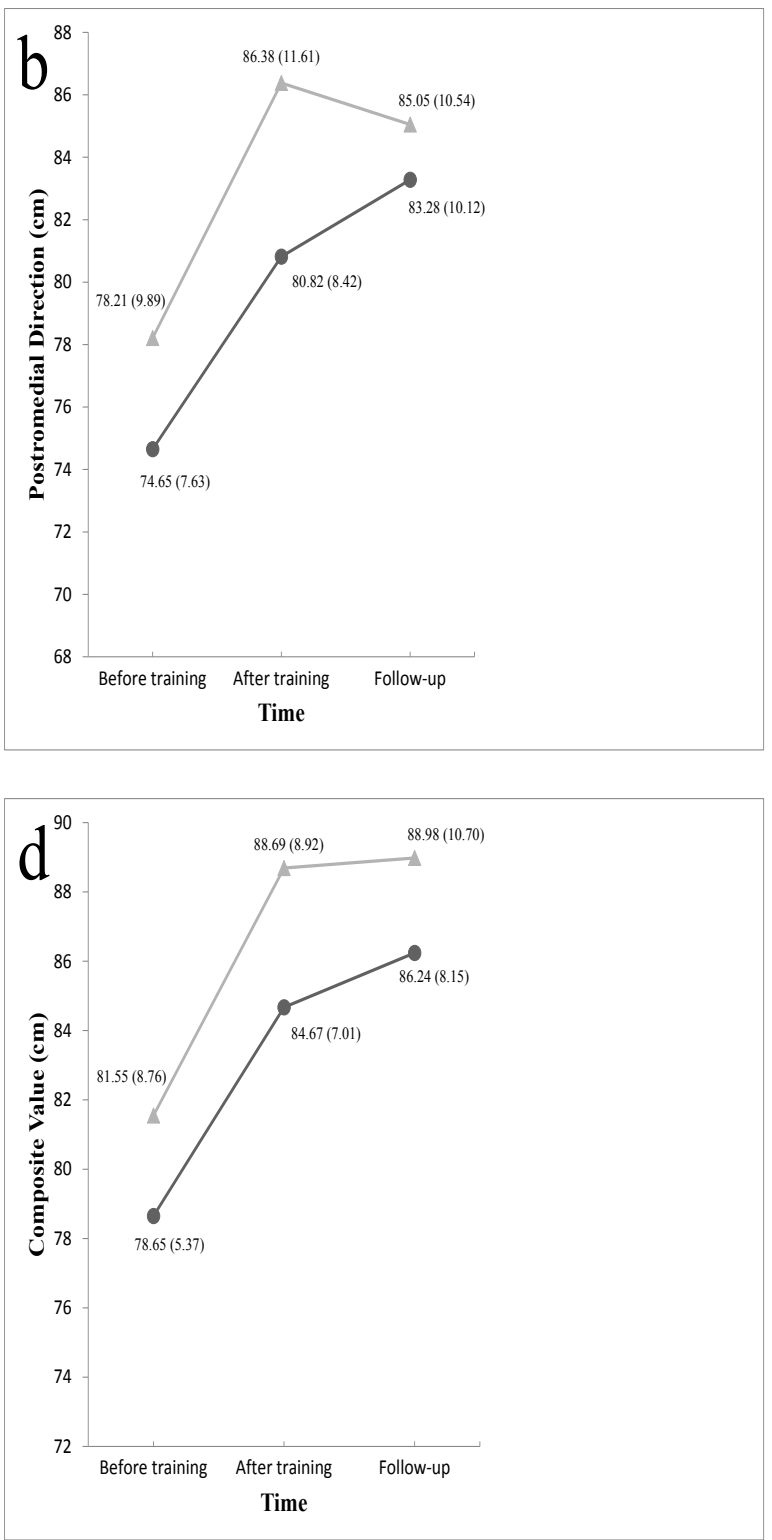

Figure 4: Group comparison of changes in dynamic balance measured with the $Y$-balance test in (a) anterior (b) posteromedial (c) posterolateral directions and (d) composite value. Values are the mean and (SD) 
ability as well as improved dynamic balance in patients with chronic NP. These effects were maintained over a 5-week follow-up period. Between-groups analyses demonstrated that VRT was more effective than CPT in reducing pain and disability. However, neither intervention was superior in improving dynamic balance.

Although both exercise protocols reduce reported pain and functional disability, VRT decreased these measures about twice as much as CPT (Figure 3). With regard to VAS scores, only VRT group showed an MCIC of $25 \mathrm{~mm}$ or more immediately after the intervention and after 5 weeks. Similarly, the improvement in NDI was reflected as an MCIC of $7 \%$ in VRT group at both time points. In CPT group, the NDI score reached MCIC value only immediately after the intervention but not after 5 weeks.

Although the mean duration of pain was $22.42 \pm 15.52$ months and baseline NP was relatively low $(47.11 \pm 10.24 \mathrm{~mm})$ in VRT group, about $75 \%$ of VAS scores and $65 \%$ of NDI scores decreased immediately after Cervigame ${ }^{\circledR}$ training and 5 -week post-intervention. The results of a previous study that evaluated the effects of another form of VRT in patients with NP demonstrated no significant improvement in pain or disability [26]. Although the participants in these two studies received similar amounts of VRT, the results are different, possibly because of differences in the VR set-up, the game itself or the study design. The results of an earlier study with 8 sessions of training with a novel method of neck coordination exercises indicated no significant improvement in VAS scores for pain. Roijezon et al. believed that this might be due to the low dosage of exercises [32]. This explanation, however, is not supported by the present study because although the dosages were similar, the results were different.

The reduction in pain and disability in our VRT group may be due to the effect of Cervigame ${ }^{\circledR}$ on deep cervical muscles. Although the activity of cervical muscles was not assessed in this study, Cervigame ${ }^{\circledR}$ appears to play an important role in the improvement of deep cervical muscle function and coordination between these and superficial muscles. These improvements may lead to better support of the cervical segments and may decrease the stress on cervical structures, which in turn would reduce NP [33].

One possible reason for the greater effectiveness of VRT than CPT in reducing pain was the unique potential of VR for pain distraction [34]. While playing the video game, the participant engages in an attention-demanding task. Because human attentional resources are finite, the participant's cognitive capacity to process pain is reduced during play, and therefore perceived pain decreases [20].

The decreases in reported pain and disability in our CPT group are consistent with previous studies [15, 16, 35]. Jull et al. believed that these effects might be due to a reduction in the interference of pain with transmission of afferent input at the subcortical or cortical levels of dorsal horn [15]. This mechanism may also be involved during our VRT protocol.

With regard to dynamic balance, the results for all directions and the composite YBT value were similar in both groups (Figure 4). There was significant YBT improvement immediately after the intervention and after 5 weeks. We found that VRT was as effective as CPT in improving dynamic balance. Earlier findings showed that neck coordination exercising improved postural control in a sample of patients with nonspecific NP [32]. The effect of CPT on dynamic balance may be due to improvements in neck proprioception acuity, as shown in previous studies $[15,16]$. This explanation may also hold for VRT, but because the effect of Cervigame ${ }^{\circledR}$ on muscle function has not yet been examined, this possibility remains speculative. Another possible explanation is the improvement in reflex connections between head, neck eye and vestibular system, and this possibility should be investigated in 
future studies.

We obtained positive results with regard to the clinical applicability of Cervigame ${ }^{\circledR}$. Almost all participants in VRT group were satisfied with the game. After the last session of VRT, some participants requested a copy of the game to continue playing on their own, but our study design precluded permission for this. There were no reports of discomfort, motion sickness or pain exacerbation during or after playing the game.

The limitations of this study should be noted. Only patients with nonspecific chronic NP and mild functional disability according to NDI score were recruited. Future studies should investigate the effectiveness of Cervigame ${ }^{\circledR}$ for rehabilitation in patients with different disorders and greater levels of disability, as well as in older populations. This study was not designed to assess the activity of superficial or deep cervical muscles or their propriceptive function, and the interpretation of our results is therefore limited. Additional work is needed to investigate the relationship between cervical muscle function and the effectiveness of the game. Finally, because designing Cervigame ${ }^{\circledR}$ was relatively time consuming and the clinical applicability of the game was initially unclear, only 50 stages of the video game have been developed to date. Nevertheless, 8 sessions of training may not have been sufficient to detect the full effects of the game. In light of the encouraging results of this study, further development of Cervigame ${ }^{\circledR}$ seems warranted.

\section{Conclusion}

VR intervention tested here significantly improved pain, functional disability and dynamic balance immediately after and 5 weeks after the intervention in patients with nonspecific chronic NP. Although similar results were seen in our CPT group, VRT was more effective in reducing pain and functional disability. Considering the level of satisfaction among participants who played Cervigame ${ }^{\circledR}$ and the absence of reported side effect, this new type of training has the potential to become part of a rehabilitation plan for patients with neck disorders. Although Cervigame ${ }^{\circledR}$ is in its infancy, the present findings suggest that efforts are warranted to develop and refine it further in the future studies.

\section{Acknowledgment}

This article reports research done for a $\mathrm{PhD}$ thesis by Iman Rezaei, and was financially supported by the Vice Chancellor for Research of Shiraz University of Medical Sciences through grant No. 92-6895. We thank K. Shashok (Author AID in the Eastern Mediterranean) for improving the use of English in the manuscript.

\section{Conflict of Interest}

None

\section{References}

1. Guez M, Hildingsson C, Nilsson M, Toolanen $G$. The prevalence of neck pain: a population-based study from northern Sweden. Acta Orthop Scand. 2002;73:455-9. doi. org/10.1080/00016470216329. PubMed PMID: 12358121.

2. Makela $M$, Heliovaara $M$, Sievers $K$, Impivaara 0 , Knekt P, Aromaa A. Prevalence, determinants, and consequences of chronic neck pain in Finland. $A m$ J Epidemiol. 1991;134:1356-67. PubMed PMID: 1755449.

3. Bovim G, Schrader H, Sand T. Neck pain in the general population. Spine (Phila Pa 1976). 1994;19:1307-9. doi.org/10.1097/00007632199406000-00001. PubMed PMID: 8066508.

4. Sjolander P, Michaelson P, Jaric S, Djupsjobacka $M$. Sensorimotor disturbances in chronic neck pain--range of motion, peak velocity, smoothness of movement, and repositioning acuity. Man Ther. 2008;13:122-31. doi.org/10.1016/j. math.2006.10.002. PubMed PMID: 17197230.

5. Tsang SM, Szeto GP, Lee RY. Movement coordination and differential kinematics of the cervical and thoracic spines in people with chronic neck pain. Clin Biomech (Bristol, Avon). 2013;28:610-7. doi. org/10.1016/j.clinbiomech.2013.05.009. PubMed PMID: 23777907.

6. Woodhouse A, Vasseljen 0. Altered motor con- 
trol patterns in whiplash and chronic neck pain. BMC Musculoskelet Disord. 2008;9:90. doi. org/10.1186/1471-2474-9-90. PubMed PMID: 18570647. PubMed PMCID: 2446396.

7. Sarig Bahat H, Chen X, Reznik D, Kodesh E, Treleaven J. Interactive cervical motion kinematics: sensitivity, specificity and clinically significant values for identifying kinematic impairments in patients with chronic neck pain. Man Ther. 2015;20:295-302. doi.org/10.1016/j.math.2014.10.002. PubMed PMID: 25456272.

8. Liu JX, Thornell LE, Pedrosa-Domellof F. Muscle spindles in the deep muscles of the human neck: a morphological and immunocytochemical study. J Histochem Cytochem. 2003;51:175-86. doi. org/10.1177/002215540305100206. PubMed PMID: 12533526.

9. Kulkarni V, Chandy MJ, Babu KS. Quantitative study of muscle spindles in suboccipital muscles of human foetuses. Neurol India. 2001;49:355-9. PubMed PMID: 11799407.

10. Treleaven J. Sensorimotor disturbances in neck disorders affecting postural stability, head and eye movement control. Man Ther. 2008;13:2-11. doi. org/10.1016/j.math.2007.06.003. PubMed PMID: 17702636

11. Bove M, Courtine G, Schieppati M. Neck muscle vibration and spatial orientation during stepping in place in humans. $J$ Neurophysiol. 2002;88:223241. doi.org/10.1152/jn.00198.2002. PubMed PMID: 12424265.

12. Kavounoudias A, Gilhodes JC, Roll R, Roll JP. From balance regulation to body orientation: two goals for muscle proprioceptive information processing? Exp Brain Res. 1999;124:80-8. doi.org/10.1007/ s002210050602. PubMed PMID: 9928792.

13. Silva $A G$, Cruz AL. Standing balance in patients with whiplash-associated neck pain and idiopathic neck pain when compared with asymptomatic participants: A systematic review. Physiother Theory Pract. 2013;29:1-18. doi.org/10.3109/09593985.2 012.677111. PubMed PMID: 22515180.

14. Jull GA, Falla D, Vicenzino B, Hodges PW. The effect of therapeutic exercise on activation of the deep cervical flexor muscles in people with chronic neck pain. Man Ther. 2009;14:696-701. doi. org/10.1016/j.math.2009.05.004. PubMed PMID: 19632880.

15. Jull G, Falla D, Treleaven J, Hodges P, Vicenzino B. Retraining cervical joint position sense: the effect of two exercise regimes. J Orthop Res. 2007;25:40412. doi.org/10.1002/jor.20220. PubMed PMID: 17143898 .
16. Revel M, Minguet M, Gregoy P, Vaillant J, Manuel JL. Changes in cervicocephalic kinesthesia after a proprioceptive rehabilitation program in patients with neck pain: a randomized controlled study. Arch Phys Med Rehabil. 1994;75:895-9. doi.org/10.1016/0003-9993(94)90115-5. PubMed PMID: 8053797.

17. McCaskey MA, Schuster-Amft C, Wirth B, Suica Z, de Bruin ED. Effects of proprioceptive exercises on pain and function in chronic neck- and low back pain rehabilitation: a systematic literature review. BMC Musculoskelet Disord. 2014;15:382. doi. org/10.1186/1471-2474-15-382. PubMed PMID: 25409985.

18. Adamovich SV, Fluet GG, Tunik E, Merians AS. Sensorimotor training in virtual reality: a review. NeuroRehabilitation. 2009;25:29-44. PubMed PMID: 19713617. PubMed PMCID: 2819065.

19. Holden MK. Virtual environments for motor rehabilitation: review. Cyberpsychol Behav. 2005;8:187211; discussion 212-9. doi.org/10.1089/ cpb.2005.8.187. PubMed PMID: 15971970.

20. Malloy KM, Milling LS. The effectiveness of virtual reality distraction for pain reduction: a systematic review. Clin Psychol Rev. 2010;30:1011-8. doi. org/10.1016/j.cpr.2010.07.001. PubMed PMID: 20691523.

21. Miller AC, Hickman LC, Lemasters GK. A distraction technique for control of burn pain. J Burn Care Rehabil. 1992;13:576-80. doi.org/10.1097/00004630199209000-00012. PubMed PMID: 1452593.

22. Li A, Montano Z, Chen VJ, Gold JI. Virtual reality and pain management: current trends and future directions. Pain Manag. 2011;1:147-57. doi. org/10.2217/pmt.10.15. PubMed PMID: 21779307. PubMed PMCID: 3138477.

23. Mahrer NE, Gold Jl. The use of virtual reality for pain control: a review. Curr Pain Headache Rep. 2009;13:100-9. doi.org/10.1007/s11916-0090019-8. PubMed PMID: 19272275.

24. Wiederhold BK, Gao K, Sulea C, Wiederhold MD. Virtual reality as a distraction technique in chronic pain patients. Cyberpsychol Behav Soc Netw. 2014;17:346-52. doi.org/10.1089/ cyber.2014.0207. PubMed PMID: 24892196. PubMed PMCID: 4043365.

25. Burdea GC. Virtual rehabilitation--benefits and challenges. Methods Inf Med. 2003;42:519-23. PubMed PMID: 14654886.

26. Sarig Bahat $H$, Takasaki $H$, Chen $X$, Bet-Or $Y$, Treleaven J. Cervical kinematic training with and without interactive VR training for chronic neck pain - a randomized clinical trial. Man Ther. 2015;20:68- 
78. doi.org/10.1016/j.math.2014.06.008. PubMed PMID: 25066503.

27. Jansen-Kosterink SM, Huis In 't Veld RM, Schonauer C, Kaufmann $\mathrm{H}$, Hermens HJ, Vollenbroek-Hutten MM. A Serious Exergame for Patients Suffering from Chronic Musculoskeletal Back and Neck Pain: A Pilot Study. Games Health J. 2013;2:299-307. doi.org/10.1089/g4h.2013.0043. PubMed PMID: 24761327. PubMed PMCID: 3833375.

28. Pool JJ, Ostelo RW, Hoving JL, Bouter LM, de Vet HC. Minimal clinically important change of the Neck Disability Index and the Numerical Rating Scale for patients with neck pain. Spine (Phila Pa 1976). 2007;32:3047-51. doi.org/10.1097/ BRS.0b013e31815cf75b. PubMed PMID: 18091500.

29. Vernon $\mathrm{H}$, Mior S. The Neck Disability Index: a study of reliability and validity. I Manipulative Physiol Ther. 1991;14:409-15. PubMed PMID: 1834753.

30. Mousavi SJ, Parnianpour M, Montazeri A, Mehdian $\mathrm{H}$, Karimi $\mathrm{A}$, Abedi M, et al. Translation and validation study of the Iranian versions of the Neck Disability Index and the Neck Pain and Disability Scale. Spine (Phila Pa 1976). 2007;32:E82531. doi.org/10.1097/BRS.0b013e31815ce6dd. PubMed PMID: 18091478.
31. Arami J, Rezasoltani A, Khalkhali Zaavieh M, Rahnama L. The effect of two exercise therapy programs (proprioceptive and endurance training) to treat patients with chronic non-specific neck pain. Journal of Babol University of Medical Sciences. 2012;14:77-84.

32. Roijezon U, Bjorklund M, Bergenheim M, Djupsjobacka M. A novel method for neck coordination exercise--a pilot study on persons with chronic nonspecific neck pain. J Neuroeng Rehabil. 2008;5:36. doi.org/10.1186/1743-0003-5-36. PubMed PMID: 19105826. PubMed PMCID: 2625342.

33. Harel NY, Song KH, Tang X, Strittmatter SM. Nogo receptor deletion and multimodal exercise improve distinct aspects of recovery in cervical spinal cord injury. J Neurotrauma. 2010;27:2055-66. doi.org/10.1089/neu.2010.1491. PubMed PMID: 20809785. PubMed PMCID: 2978056.

34. Gold JI, Belmont KA, Thomas DA. The neurobiology of virtual reality pain attenuation. Cyberpsychol Behav. 2007;10:536-44. doi.org/10.1089/ cpb.2007.9993. PubMed PMID: 17711362.

35. Humphreys B, Irgens $P$. The effect of a rehabilitation exercise program on head repositioning accuracy and reported levels of pain in chronic neck pain subjects. Journal of Whiplash \& Related Disorders. 2002;1:99-112. doi.org/10.3109/ j180v01n01_09. 